\title{
A UTILIZAÇÃO DA ACUPUNTURA EM SINAIS E SINTOMAS DAS DISFUNÇÕES TEMPOROMANDIBULARES: REVISÃO SISTEMÁTICA
}

\author{
Deborah Santos Morgado da Costa ${ }^{1}$ \\ Nathalia Winkler ${ }^{2}$ \\ Leandro Júnio Masulo ${ }^{3}$ \\ Renata Amadei Nicolau ${ }^{4}$ \\ Josne Carla Paterno ${ }^{5}$
}

\begin{abstract}
Resumo- $A$ acupuntura é uma técnica terapêutica da Medicina Tradicional Chinesa. Os efeitos da acupuntura são: diminuição da dor e o relaxamento muscular pela utilização de agulhas. As Disfunções Temporomandibulares (DTMs) afetam o sistema estomatognático, envolvendo estruturas ósseas, musculares e na região orofacial. Foram coletados 11 artigos publicados dos últimos 10 anos (2005-2015) através das bases Scielo, Bireme e Google Acadêmico. 64\% foram realizados com seres humanos. A acupuntura para dor em DTM é instituída durante aproximadamente 5 sessões de 18 minutos, normalmente 1 vez por semana. Os métodos de avaliação da dor mais citados são: Escala Visual Analógica (42\%) onde deste percentual, 17\% relatam Índice de Disfunção Craniomandibular associado à Escala Visual Analógica e 8\% relatam o Exame clínico com associação da Escala Visual Analógica. 8\% relatam somente o Exame clínico, Research Diagnostic Criteria for Temporomandibular Disorders (8\%) e Índice de Disfunção Craniomandibular (8\%). Conclui-se que a acupuntura é uma terapia efetiva, que traz como principais benefícios o relaxamento e diminuição da dor muscular em pacientes com DTMs.
\end{abstract}

Palavras-chave: Acupuntura; Articulação;Temporomandibular; Odontologia.

\footnotetext{
1 Odontologia/Universidade do Vale do Paraíba, Brasil. E-mail: debbysmc@yahoo.com.br.

2 Odontologia/Universidade do Vale do Paraíba, Brasil. E-mail: nathaliawinkler@hotmail.com.

3 Odontologia/Universidade do Vale do Paraíba, Brasil. E-mail: Imasulo@univap.br.

4 Odontologia/Universidade do Vale do Paraíba, Brasil. E-mail: rani@univap.br.

${ }^{5}$ Biomedicina/Universidade Federal de São Paulo, Brasil. E-mail: josneparterno@gmail.com.
} 